\title{
Perceptions of cultural competency of undergraduate nursing students*
}

\author{
Diane Von Ah, Natasha Cassara \\ Adult Health Department, Indiana University School of Nursing, Indianapolis, USA \\ Email: dvonah@iupui.edu
}

Received 26 February 2013; revised 30 March 2013; accepted 16 April 2013

Copyright (C) 2013 Diane Von Ah, Natasha Cassara. This is an open access article distributed under the Creative Commons Attribution License, which permits unrestricted use, distribution, and reproduction in any medium, provided the original work is properly cited.

\begin{abstract}
Purpose: The purpose of this study was to examine the level of cultural competence of undergraduate nursing students at a large Midwestern University. Background: Developing cultural competence of nurses is critical to meet the needs of our growing diverse society. Methods: A convenience sample of 150 undergraduate nursing students completed a one-time questionnaire assessing students' cultural competence. Results: Nursing students' rated their overall knowledge and comfort moderate to poor with only $28 \%$ rated themselves as very comfortable and $15 \%$ as very skillful in providing culturally competent care. Students rated their knowledge and comfort highest in taking a family history, whereas, the lowest scores were related to pregnancy and childbirth, death and dying, and organ donation in other cultures. Conclusions: Findings suggest that nursing students feel inadequately prepared to provide culturally competent care.
\end{abstract}

Keywords: Cultural Diversity; Cultural Competency; Nursing Education; Cultural Self-Efficacy

\section{INTRODUCTION}

Cultural diversity is a significant issue to address in nursing curriculum. The United States (US) is rapidly becoming a more diverse nation. More than one-third of the US population identify themselves as a "minority" (other than Non-Hispanic White), a jump of $11 \%$ from 2000 [1]. In fact, it is estimated that by 2050, members of culturally and ethnically diverse populations will comprise $50 \%$ of the US population [2]. Conversely, the nursing

\footnotetext{
*The authors would like to acknowledge funding from Robert Wood
} Johnson Foundation, Nurse Faculty Scholar Grant (PI: Von Ah, \#64194). profession continues to be disproportionally represented by non-Hispanic, White individuals [3]. The concern for lack of diversity in nursing was highlighted in the institutes of medicine report titled, "The Future of Nursing: Leading Health, Advancing Change”, which calls for both a "greater emphasis on increasing diversity of the workforce and ensuring that nurses are able to provide culturally relevant care" [4].

\section{BACKGROUND}

\subsection{Nursing Education}

Nursing programs have a responsibility to adequately prepare their graduates to provide culturally competent care. The Essentials of Baccalaureate Education for Professional Nursing Practice emphasizes cultural competence in several of the outcome competencies for nursing graduates [5]. In addition, multiple organizations and educators have offered direction in developing cultural competency in nursing curriculum [6,7]. With the growing emphasis, the purpose of this study was to examine undergraduate nursing student's perception of cultural competence at a major Midwestern University School of Nursing.

\subsection{Conceptual Framework}

Cultural competence has been defined as, "the routine application of culturally appropriate health care interventions and practices” [2, p. 193]. The Cultural Development Model developed by Wells, conceptualizes that two separate phases are needed in the development of cultural competence: the cognitive (knowledge) and the affective (attitude and behavior) phase [2]. During the cognitive phase emphasis is placed on learning and acquiring knowledge about culture. The affective phase focuses on attitudinal and behavioral change through the 
application of the knowledge acquired in the cognitive phase. Cultural competence, then, is an ongoing process requiring more than just formal knowledge. Values and attitudes must change to form the foundation for the commitment to providing culturally competent care [2].

\section{METHODS}

\subsection{Design and Sample}

A descriptive cross-sectional design was used to examine the level of cultural competence of undergraduate nursing students. Nursing students were eligible if they were enrolled and registered for nursing core courses at a major Midwestern University, which at the time of the study had over 700 undergraduate students (522 traditional and 182 accelerated students) enrolled.

\subsection{Procedure}

A one-time electronic questionnaire was sent to undergraduate nursing students enrolled at a large Midwestern University to assess student's perceived knowledge, attitudes, and comfort skills regarding cultural competence. The purpose of the study, risks and benefits of participation, requirements of participation, and survey questionnaires with instructions were sent to potential participants. Completion of the survey instruments was denoted as consent to participate in this study. A one month follow-up electronic message to participate was sent to all potential participants to improve the response rate. Students did not receive an incentive to participate.

The University Institutional Review Board granted approval for the study.

\subsection{Instrument}

Cultural Competence was measured by the Caffrey Cultural Competence in Healthcare Scale (CCCHS) which was based on Well's model [8]. The CCCHS is a 28-item 5-point Likert self-rating scale which measures the respondents' cultural immersion (e.g., fluency in a second language, time abroad, and contact with other cultural groups) and self-perceived knowledge, self-awareness, and comfort with skills of cultural competence; with higher scores indicating higher cultural competence. Cronbach's coefficient alpha in this study was 0.93 .

\subsection{Data Analysis}

Data were analyzed using SPSS ${ }^{\circledR} 13.0$ for Windows (SPSS Inc., Chicago, IL). Descriptive statistics and analysis of variance (ANOVA) was used to describe the sample and to determine the level of overall perceived cultural competence and differences by grade level (sophomores, juniors, seniors, and accelerated).

\section{RESULTS}

\subsection{Sample}

150 undergraduate nursing students including sophomores ( $n=55$; 33.3\%); juniors ( $n=39 ; 23.6 \%$ ), seniors ( $n=35 ; 21.3 \%)$, and accelerated nursing students $(n=36$; $21.8 \%$ ) participated. Students ranged in age from 18 to 57 with a mean age of 25 . The majority of the students (95\%) were female and identified themselves as White non-Hispanic (87\%) and $94 \%$ of respondents identified English as their primary language.

\subsection{Cultural Experience}

First the students self-identified their level of immersion in another culture. From this data we noted that only $22 \%$ of students indicated that they had spent a month or longer in a country other than the US. Similarly, only $8.5 \%$ of students reported that they had a great deal of contact with patients from another culture than their own and $9 \%$ felt they had a great deal of contact with healthcare workers from another culture.

\subsection{Knowledge and Comfort of Cultural Competence}

The average overall rating of the nursing students' knowledge, self-awareness and comfort regarding cultural competence was 3.34, $(\mathrm{SD}=0.66)$ on a 5-point scale. No significant differences were noted between the different grade levels on overall knowledge and comfort regarding cultural competence. The sophomores' overall score was rated $\mathrm{M}=3.28$ ( $\mathrm{SD}=1.04$ ), juniors' was $\mathrm{M}=3.26$ ( $\mathrm{SD}=$ 1.02), seniors' was $\mathrm{M}=3.46$ ( $\mathrm{SD}=0.94)$, and accelerated students' was $\mathrm{M}=3.40(\mathrm{SD}=0.95)$ on a 5 -point Likert scale.

While the overall scores on the CCCHS were moderate, there were definite areas in which the students rated their knowledge, self-awareness and comfort with skills in cultural competency as considerably higher or lower than others. Students rated their comfort level highest in working with a translator $(\mathrm{M}=4.26, \mathrm{SD}=0.91)$ and working as a team member with health care providers from a cultural group other than their own ( $\mathrm{M}=4.21$, SD $=0.90)$. The lowest scores were noted in knowledge with practices in other cultures related to death and dying (M $=2.85, \mathrm{SD}=1.01)$, pregnancy and childbirth $(\mathrm{M}=2.79$, $\mathrm{SD}=1.03)$, and organ donation $(\mathrm{M}=2.37, \mathrm{SD}=1.02)$. In addition, only $28 \%$ of students perceived themselves as very comfortable and $15 \%$ as very skillful in their abilities in providing culturally competent care.

\section{DISCUSSION}

Our results indicate that, although there has been an em- 
phasis for incorporating cultural competence in nursing programs, nursing students do not feel confident in providing culturally competent care. These findings are consistent with a much earlier integrative review (including 5 studies and a combined total of 620 nursing students) in which undergraduate nursing students were not confident in caring for ethnically diverse patient populations [9]. Together, these findings indicate that little has changed since the implementation of the Essentials of Baccalaureate Education for Professional Nursing Practice (American Association of Colleges of Nursing, 2008). Nursing programs must do more to prepare nursing students to meet the needs of culturally diverse patients $[1,10,11]$.

This work, to our knowledge, was also the first to identify specific areas of strengths and weaknesses perceived by undergraduate nursing students. Nursing faculty should build on perceived strengths but, more importantly focus on areas of greater concern for undergraduates. Students rated their knowledge, attitude and skill highest in working with a translator and working as a team member with health care providers from a cultural group other than their own, whereas they rated their knowledge, attitude and skill lowest with practices in other cultures related to pregnancy and childbirth, death and dying, and organ donation. These topics are often difficult for nursing students and new nurses but can be even more difficult when working with individuals from other cultures. In the nursing literature, we noted eight intervention studies to improve cultural competence among undergraduate nursing students [8,12-18]. These studies used a variety of teaching modalities including lectures, role-play, web-based interaction, journaling, case-studies, and service-based learning. Our findings suggest that interventions, such as those outlined in these studies, may be enhanced by adding a needs-based approach within the curriculum to assess and target students' greatest areas of concern.

Notably, few of the nursing students participating in our study had significant cultural immersion experience. This lack of exposure may have impacted the results of our study. Coffman and colleagues (2004) noted that exposure to ethnically diverse populations was associated with increased cultural self-efficacy. Larsen and Rief (2011) noted that nursing students who participated in a 2- to 3-week immersion program rated their transcultural self-efficacy significantly greater than those students who were in the control group. Similarly, Kuehn et al., (2011) conducted a study with undergraduate nursing students from the United States, Mexico, and Canada who were provided with intercultural experiences online which included discussions regarding nursing care and health care norms and issues in their different countries. Participating students reported that talking with students from other countries, especially through live online chats, enabled them to discuss and learn about issues that had never been brought to their attention (2011). These findings support the importance of creating opportunities for immersion in diverse populations of patients either directly or indirectly through the use of interactive technology.

\subsection{Implications}

The findings of this study have important implications for nursing programs whose role it is to prepare culturally competent nursing students. In addition, the results may also impact healthcare agencies that employ nurses to care for their culturally diverse populations. Nursing schools need to take a multifaceted approach to implementing cultural competence in their standard program. A cultural assessment tool to evaluate student's knowledge, skills, and attitudes throughout the nursing program could be used to enhance the existing curriculum and address the on-going needs of students. Facilitating conversations between nursing students from other countries through live online chats may also help improve our students' knowledge and attitudes towards topics such as pregnancy, death, organ donation, and other significant issues that may not be adequately addressed in the nursing curriculum. The provision of culturally competent care is extremely important as it may help reduce disparities in the health status of Americans by allowing equitable, high-quality healthcare to all patients regardless of race, culture, or socioeconomic status [1].

\subsection{Limitations}

There were several limitations for this study. The main issue centers on the sampling. A convenience sample was used and only about a quarter of the students (22\%) participated in this one-time survey. Thus, those who responded may have been more interested in cultural competence which would impact study findings. In addition, this was a cross sectional survey and more information may have been derived by examining the level of competence for each student over time (from the beginning to the completion of the nursing program).

\section{CONCLUSION}

The findings of this study support the importance of adequately addressing cultural competence in the undergraduate nursing curriculum by providing students with the knowledge and clinical experiences that will enable them to be comfortable and sensitive to the needs of diverse patient populations. Developing cultural competence of nurses is critical if nurses are to respond effectively to the needs of our growing diverse society. Further research is needed among schools of nursing to as- 
sess the knowledge, attitudes, and skills of undergraduate nursing students to ensure that they are preparing nurses who are culturally competent to care for diverse populations of patients.

\section{REFERENCES}

[1] Onyoni, E. and Ives, T. (2007) Assessing implementation of cultural competency content in the curricula of colleges of pharmacy in the United States and Canada. American Journal of Pharmaceutical Education, 71, 1-7. doi:10.5688/aj710224

[2] Wells, M.I. (2000) Beyond cultural competence: A model for individual and institutional cultural development. Journal of Community Health, 17, 189-199. doi:10.1207/S15327655JCHN1704 1

[3] Sanner, S., Baldwin, D., Cannella, K., Charles, J. and Parker, L. (2010) The impact of cultural diversity forum on students' openness to diversity. Journal of Cultural Diversity, 17, 56-61.

[4] Institute of Medicine (2011) The future of nursing: Leading change, advancing health. The National Academies Press, Washington DC.

[5] American Association of Colleges of Nursing (AACN) (2008) The essentials of baccalaureate education for professional nursing practice. American Association of Colleges of Nursing, Washington DC.

[6] Cuellar, N.G., Walsh Brennan, A.M., Vito, K. and De Leon Siantz, M.L. (2008) Cultural competency in the undergraduate nursing curriculum. Journal of Professional Nursing, 24, 43-49. doi:10.1016/j.profnurs.2008.01.004

[7] Giger, J., Davidhizar, R.E., Purnell, L., Harden, J.T., Phillips, J., Strickland, O. and American Academy of Nursing (2007) American academy of nursing expert panel report: Developing cultural competence to eliminate health disparities in ethnic minorities and other vulnerable populations. Journal of Transcultural Nursing, 18, 95-102.

[8] Caffrey, R.A., Neander, W., Markle, D. and Stewart, B. (2005) Improving the cultural competence of nursing students: Results of integrating cultural content in the curriculum and an international immersion experience. Journal of Nursing Education, 44, 234-240.
[9] Coffman, M.J., Shellman, J. and Bernal, H. (2004) An integrative review of American nurses' perceived cultural self-efficacy. Journal of Nursing Scholarship, 36, 180185. doi:10.1111/j.1547-5069.2004.04032.x

[10] Kardong-Edgren, S., Cason, C.L., Brennan, A.M.W., Reifsnider, E., Hummel, F., Mancini, M. and Griffin, C. (2010) Cultural competency of graduating BSN nursing students. Nursing Education Perspectives, 31, 278-285.

[11] Kennedy, H., Fisher, L., Fontaine, D. and Martin-Holland, J. (2008) Evaluating diversity in nursing education: A mixed method study. Journal of Transcultural Nursing, 19, 363-370. doi:10.1177/1043659608322500

[12] Amerson, R. (2010) The impact of service-learning on cultural competence. Nursing Education Research, 13, 1822.

[13] Hughes, K.H. and Hood, L.J. (2007) Teaching methods and an outcome tool for measuring cultural sensitivity in undergraduate students. Journal of Transcultural Nursing, 18, 167-174.

[14] Musolino, G.M., Babitz, M., Burkalter, S.T., Thompson, C., Harris, R., Ward, R.S. and Chase-Cantarini, S. (2009) Mutual respect in healthcare: Assessing cultural competence for the University of Utah interdisciplinary health sciences. Journal of Allied Health, 38, 54-62.

[15] Napholz, L. (1999) A comparison of self-reported cultural competency skills among two groups of nursing students: Implications for nursing education. Journal of Nursing Education, 38, 81-83.

[16] Nokes, K.M., Nickitas, D.M., Keida, R. and Neville, S. (2005) Does service-learning increase cultural competency, critical thinking, and civic engagement. Journal of Nursing Education, 44, 65-70.

[17] Larsen, R. and Rief, L. (2011) Effectiveness of cultural immersion and cultural classes for enhancing students' transcultural self-efficacy. Journal of Nursing Education, 50, 350-354.

[18] Kuehn, A.F., Chircop, A., Downe-Wambolt, B. SheppardLeMoine, D., Wittock, L., Herbert, R., Benavides Torres, R.A., Murnaghan, D. and Critchly, K. (2011) Evaluating the impact of North American research exchange program on student cultural awareness. International Journal of Nursing Education Scholarship, 8, 1-15. doi:10.2202/1548-923X.1919 